\title{
MULTIPLE LATTICE TILES AND RIESZ BASES OF EXPONENTIALS
}

\author{
MIHAIL N. KOLOUNTZAKIS
}

(Communicated by Alexander Iosevich)

\begin{abstract}
Suppose $\Omega \subseteq \mathbb{R}^{d}$ is a bounded and measurable set and $\Lambda \subseteq \mathbb{R}^{d}$ is a lattice. Suppose also that $\Omega$ tiles multiply, at level $k$, when translated at the locations $\Lambda$. This means that the $\Lambda$-translates of $\Omega$ cover almost every point of $\mathbb{R}^{d}$ exactly $k$ times. We show here that there is a set of exponentials $\exp (2 \pi i t \cdot x), t \in T$, where $T$ is some countable subset of $\mathbb{R}^{d}$, which forms a Riesz basis of $L^{2}(\Omega)$. This result was recently proved by Grepstad and Lev under the extra assumption that $\Omega$ has boundary of measure 0 , using methods from the theory of quasicrystals. Our approach is rather more elementary and is based almost entirely on linear algebra. The set of frequencies $T$ turns out to be a finite union of shifted copies of the dual lattice $\Lambda^{*}$. It can be chosen knowing only $\Lambda$ and $k$ and is the same for all $\Omega$ that tile multiply with $\Lambda$.
\end{abstract}

Notation. We write $e(x)=e^{2 \pi i x}$. If $E$ is a set, then $\chi_{E}$ is its indicator function. If $A$ is a non-singular $d \times d$ matrix and $\Lambda=A \mathbb{Z}^{d}$ is a lattice in $\mathbb{R}^{d}$, then $\Lambda^{*}=A^{-\top} \mathbb{Z}^{d}$ denotes the dual lattice.

\section{INTRODUCTION}

1.1. Riesz bases. In this paper we deal with the question of the existence of a Riesz (unconditional) basis of exponentials

$$
e_{t}(x):=e(t \cdot x)=e^{2 \pi i t \cdot x}, \quad t \in L,
$$

for the space $L^{2}(\Omega)$, where $\Omega \subseteq \mathbb{R}^{d}$ is a domain of finite Lebesgue measure and $L \subseteq \mathbb{R}^{d}$ is a countable set of frequencies. By Riesz basis we mean that every $f \in L^{2}(\Omega)$ can be written uniquely in the form

$$
f(x)=\sum_{t \in L} a_{t} \cdot e(t \cdot x)
$$

with the coefficients $a_{t} \in \mathbb{C}$ satisfying

$$
C_{1}\|f\|_{2}^{2} \leq \sum_{t \in L}\left|a_{t}\right|^{2} \leq C_{2}\|f\|_{2}^{2}
$$

for some positive and finite constants $C_{1}, C_{2}$.

Received by the editors May 12, 2013.

2010 Mathematics Subject Classification. Primary 42B99.

Key words and phrases. Riesz bases of exponentials, tiling.

The author was supported in part by grant No 3803 from the University of Crete. 
1.2. Orthogonal bases. One very special example of a Riesz basis occurs when the exponentials $e(t \cdot x), t \in L$, can be chosen to be orthogonal and complete for $L^{2}(\Omega)$. One can then choose $a_{t}=|\Omega|^{-1 / 2}\left\langle f, e_{t}\right\rangle$ and $C_{1}=C_{2}=1 /|\Omega|$ for (2) to hold as an equality. For instance, if $\Omega=(0,1)^{d}$ is the unit cube in $\mathbb{R}^{d}$, then one can take $L=\mathbb{Z}^{d}$ and obtain such an orthogonal basis of exponentials. This case, where an orthogonal basis of exponentials exists, is a very rigid situation though and many "reasonable" domains do not have such a basis (a ball is one example [4,11], or any other smooth convex body or any non-symmetric convex body [7]).

The problem of which domains admit an orthogonal basis of exponentials has been studied intensively. The so-called Fuglede or Spectral Set Conjecture [4] (claiming that for $\Omega$ to have such a basis it is necessary and sufficient that it can tile space by translations) was eventually proved to be false in dimension at least 3 2, 3, 9, 12, 20, in both directions. Yet the conjecture may still be true in several important special cases such as convex bodies [8], and it generated many interesting results even after the disproof of its general validity (a rather dated account may be found in [11]).

It is expected that the existence of a Riesz basis for a domain $\Omega$ is a much more general, and perhaps even generic, phenomenon, although proofs of existence of a Riesz basis for specific domains are still rather rare, especially in higher dimension [13, 14, 16. Also no domain is known not to have a Riesz basis of exponentials [13].

1.3. Lattice tiles. One general class of domains for which an orthogonal basis of exponentials is known to exist is the class of lattice tiles. A domain $\Omega \in \mathbb{R}^{d}$ is said to tile space when translated at the locations of the lattice $L$ (a discrete additive subgroup of $\mathbb{R}^{d}$ containing $d$ linearly independent vectors) if

$$
\sum_{t \in L} \chi_{\Omega}(x-t)=1, \text { for almost all } x \in \mathbb{R}^{d} .
$$

Intuitively this condition means that one can cover $\mathbb{R}^{d}$ with the $L$-translates of $\Omega$, with no overlaps, except for a set of measure zero (usually the translates of $\partial \Omega$, for "nice" domains $\Omega$ ).

It is not hard to see that when $\Omega$ has finite and non-zero measure the set $L$ has density equal to $1 /|\Omega|$. If $L$ is a lattice, then we call $\Omega$ an almost fundamental domain of $L$ and $|\Omega|=(\operatorname{dens} L)^{-1}$. A fundamental domain of $L$ is any set which contains exactly one element of each coset $\bmod L$, for instance a fundamental parallelepiped. There are of course many others, as indicated in Figure 1.

It is easy to see [4,11 that every lattice tile by the lattice $L$ has an orthogonal basis of exponentials, namely those with frequencies $t \in L^{*}$, where $L^{*}$ is the dual lattice.

1.4. Multiple tiling by a lattice. We say that a domain tiles multiply when its translates cover space the same number of times, almost everyhwere.

Definition 1.1. Let $\Omega \subseteq \mathbb{R}^{d}$ be measurable and $L \subseteq \mathbb{R}^{d}$ be a countable set. We say that $\Omega$ tiles $\mathbb{R}^{d}$ when translated by $L$ at level $k \in \mathbb{N}$ if

$$
\sum_{t \in L} \chi_{\Omega}(x-t)=k
$$

for almost every $x \in \mathbb{R}^{d}$. If we do not specify $k$, then we mean $k=1$. 


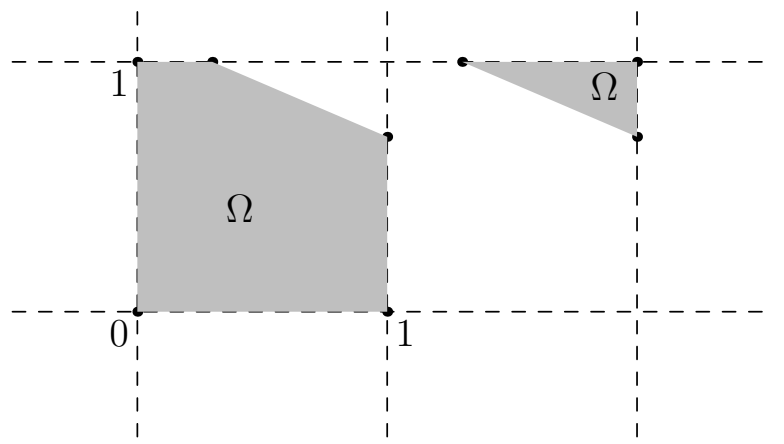

Figure 1 . Shaded $\Omega$ is a fundamental domain of $\mathbb{R}^{2} / \mathbb{Z}^{2}$

Multiple tiles are a much wider class of domains than level-one tiles. For instance [1,10, any centrally symmetric convex polygon in the plane whose vertices have integer coordinates tiles multiply by the lattice $\mathbb{Z}^{2}$ at some level $k \in \mathbb{N}$. In contrast, only parallelograms or symmetric hexagons can tile at level one.

Another difference is the fact that if two disjoint domains $\Omega_{1}$ and $\Omega_{2}$ both tile multiply when translated at the locations $L$, then so does their union. In the case of multiple lattice tiling this operation gives essentially the totality of multiple tiles starting from level-one tiles, according to the following easy lemma.

Lemma 1. Suppose $\Omega \subseteq \mathbb{R}^{d}$ is a measurable set which tiles $\mathbb{R}^{d}$ at level $k$ when translated by the lattice $\Lambda \subseteq \mathbb{R}^{d}$. Then we can partition

$$
\Omega=\Omega_{1} \cup \cdots \cup \Omega_{k} \cup E,
$$

where $E$ has measure 0 and the $\Omega_{j}$ are measurable, mutually disjoint and each $\Omega_{j}$ is an almost fundamental domain of the lattice $\Lambda$.

Proof. Let $D \subseteq \mathbb{R}^{d}$ be a measurable fundamental domain of $\Lambda$, for instance one of its fundamental parallelepipeds. For almost every $x \in D$ (call the exceptional set $E \subseteq D)$ it follows from our tiling assumption that $\Omega \cap(x+\Lambda)$ contains exactly $k$ points, which we denote by

$$
p_{1}(x)<p_{2}(x)<\cdots<p_{k}(x),
$$

ordered according to the lexicographical ordering in $\mathbb{R}^{d}$. We also have that almost every point of $\Omega$ belongs to exactly one such list.

Let then $\Omega_{j}=\bigcup_{x \in D \backslash E} p_{j}(x)$, for $j=1,2, \ldots, k$. In other words, for (almost) each one of the classes $\bmod \Lambda$ we distribute its $k$ occurences in $\Omega$ into the sets $\Omega_{j}$. It is easy to see that the $\Omega_{j}$ are disjoint and measurable and that they are almost fundamental domains of $\Lambda$.

1.5. Multiple lattice tiles have Riesz bases of exponentials. It is not true that domains that tile multiply by a lattice have an orthogonal basis of exponentials. For instance, it is known [8] that the only convex polygons that have such a basis are parallelograms and symmetric hexagons, yet every symmetric convex polygon with integer vertices is a multiple tile, a much wider class.

It is however true that multiple tiles have a Riesz basis of exponentials. The main result of this paper is the following theorem. 
Theorem 1. Suppose $\Omega \subseteq \mathbb{R}^{d}$ is bounded, measurable and tiles $\mathbb{R}^{d}$ multiply at level $k$ with the lattice $\Lambda$. Then there are vectors $a_{1}, \ldots, a_{k} \in \mathbb{R}^{d}$ such that the exponentials

$$
e\left(\left(a_{j}+\lambda^{*}\right) \cdot x\right), j=1,2, \ldots, k, \lambda^{*} \in \Lambda^{*}
$$

form a Riesz basis for $L^{2}(\Omega)$.

The vectors $a_{1}, \ldots, a_{k}$ depend on $\Lambda$ and $k$ only, not on $\Omega$.

Theorem 10was proved by Grepstad and Lev [6] with the additional topological assumption that the boundary $\partial \Omega$ has Lebesgue measure 0 .

In [6] the result is proved following the method of [17, 18] on quasicrystals. Our approach is more elementary and almost entirely based on linear algebra. The authors of 6 have pointed out that there are similarities in the method of this paper and the methods in [14 16. The method essentially appears also in [19, §3.2].

As an interesting corollary of Theorem 1 let us mention, as is done in [6], that, according to the recent result of $[5]$, if $\Omega$ is a centrally symmetric polytope in $\mathbb{R}^{d}$, whose codimension 1 faces are also centrally symmetric and whose vertices all have rational coordinates, then $L^{2}(\Omega)$ has a Riesz basis of exponentials.

Open Problem 1. Is Theorem 1 still true if $\Omega$ is of finite measure but unbounded?

\section{Proof of the MAIN RESUlT}

The essence of the proof is contained in the following lemma.

Lemma 2. Suppose $\Omega \subseteq \mathbb{R}^{d}$ is bounded, measurable and tiles $\mathbb{R}^{d}$ multiply at level $k$ with the lattice $\Lambda$. Then there exist vectors $a_{1}, a_{2}, \ldots, a_{k} \in \mathbb{R}^{d}$ such that the following is true.

For any $f \in L^{2}(\Omega)$ there are unique measurable functions $f_{j}: \mathbb{R}^{d} \rightarrow \mathbb{C}$ such that

(1) The $f_{j}$ are $\Lambda$-periodic.

(2) The $f_{j}$ are in $L^{2}$ of any almost fundamental domain of $\Lambda$.

(3) We have the decomposition

$$
f(x)=\sum_{j=1}^{k} e\left(a_{j} \cdot x\right) f_{j}(x), \quad \text { for a.e. } x \in \Omega .
$$

Finally we have

$$
C_{1}\|f\|_{L^{2}(\Omega)}^{2} \leq \sum_{j=1}^{k}\left\|f_{j}\right\|_{L^{2}(\Omega)}^{2} \leq C_{2}\|f\|_{L^{2}(\Omega)}^{2},
$$

where $0<C_{1}, C_{2}<\infty$ do not depend on $f$.

Proof. Using Lemma 1 we can write $\Omega$ as the disjoint union

$$
\Omega=\Omega_{1} \cup \cdots \cup \Omega_{k},
$$

where each $\Omega_{k}$ is a measurable almost fundamental domain of $\Lambda$. We can now define for $j=1,2, \ldots, k$ and for almost every $x \in \mathbb{R}^{d}$

$$
\omega_{j}(x) \text { as the unique point in } \Omega_{j} \text { s.t. } x-\omega_{j}(x) \in \Lambda \text {, and }
$$

$$
\lambda_{j}(x)=x-\omega_{j}(x) .
$$

(The maps $\omega_{j}$ are clearly measurable and measure-preserving when restricted to a fundamental domain of $\Lambda$.) Since the sought-after $f_{j}$ are to be $\Lambda$-periodic it is 
enough to define them on $\Omega_{1}$ and extend them to $\mathbb{R}^{d}$ by their $\Lambda$-periodicity. We may therefore rewrite our target decomposition (7) equivalently as follows.

(11)

For each $x \in \Omega_{1}$ and $r=1,2, \ldots, k: f\left(\omega_{r}(x)\right)=\sum_{j=1}^{k} e\left(a_{j} \cdot\left(x-\lambda_{r}(x)\right)\right) f_{j}(x)$.

We view (11) as a $k \times k$ linear system

$$
M \widetilde{F}=F
$$

whose right-hand side is the column vector

$$
F=\left(f\left(\omega_{1}(x)\right), f\left(\omega_{2}(x)\right), \ldots, f\left(\omega_{k}(x)\right)\right)^{\top}
$$

and the unknowns form the column vector

$$
\widetilde{F}=\left(f_{1}(x), f_{2}(x), \ldots, f_{k}(x)\right)^{\top} .
$$

We have a different linear system for each $x \in \Omega_{1}$ and its matrix is $M=M(x) \in$ $\mathbb{C}^{k \times k}$ with

$$
M_{r, j}=M_{r, j}(x)=e\left(a_{j} \cdot\left(x-\lambda_{r}(x)\right)\right), \quad r, j=1,2, \ldots, k .
$$

Factoring we can write this matrix as

$$
M(x)=N(x) \operatorname{diag}\left(e\left(a_{1} \cdot x\right), e\left(a_{2} \cdot x\right), \ldots, e\left(a_{k} \cdot x\right)\right),
$$

with the matrix $N=N(x)$ given by

$$
N_{r, j}=N_{r, j}(x)=e\left(-a_{j} \cdot \lambda_{r}(x)\right), \quad r, j=1,2, \ldots, k .
$$

The key observation here is that when varying $x \in \Omega_{1}$ the number of different $N(x)$ matrices that arise (the $a_{j}$ are fixed) is finite and bounded by a quantity that depends on $\Omega$ and $\Lambda$ only. The reason for this is that the vectors $\lambda_{r}(x)$ are among the $\Lambda$ vectors in the bounded set $\Omega-\Omega$, hence they take values in a finite set. (This is the only place where the boundedness of $\Omega$ is used.)

Let us now see that the vectors $a_{1}, \ldots, a_{k}$ can be chosen so that all the (finitely many) possible matrices $N$ are invertible. We have

$$
\operatorname{det} N(x)=\sum_{\pi \in S_{k}} \operatorname{sgn}(\pi) e\left(-\sum_{j=1}^{k} a_{j} \cdot \lambda_{\pi_{j}}(x)\right),
$$

where $S_{k}$ denotes the permutation group on $\{1,2, \ldots, k\}$. By the definition of the vectors $\lambda_{r}(x)$ and the disjointness of the sets $\Omega_{r}$ it follows that for each $x$ no two $\lambda_{r}(x)$ can be the same. View now the expression (15) as a function of the vector $a=\left(a_{1}, \ldots, a_{k}\right) \in \mathbb{R}^{d k}$. Clearly it is a trigonometric polynomial and it is not identically zero as all the frequencies (for $\pi$ in the symmetric group $S_{k}$ )

$$
\lambda_{\pi}(x)=\left(\lambda_{\pi_{1}}(x), \ldots, \lambda_{\pi_{k}}(x)\right) \in \mathbb{R}^{d k},
$$

are distinct precisely because all the $\lambda_{r}(x)$ are distinct. Since the zero-set of any trigonometric polynomial (that is not identically zero) is a set of codimension at least 1 it follows that the vectors $a_{1}, \ldots, a_{k}$ can be chosen so that all the $N(x)$ matrices that arise are invertible.

Now let $x \in \Omega_{1}$ and consider the solution of the linear system (12) at $x$ that now takes the form

$$
\widetilde{F}(x)=\operatorname{diag}\left(e\left(-a_{1} \cdot x\right), e\left(-a_{2} \cdot x\right), \ldots, e\left(-a_{k} \cdot x\right)\right) N(x)^{-1} F(x) .
$$


Since $N(x)$ runs through a finite number of invertible matrices and the diagonal matrix in (17) is an isometry it follows that there are finite constants $A_{1}, A_{2}>0$, independent of $f$, such that for any $x \in \Omega_{1}$ we have

$$
A_{1}\|F(x)\|_{\ell^{2}}^{2} \leq\|\widetilde{F}(x)\|_{\ell^{2}}^{2} \leq A_{2}\|F(x)\|_{\ell^{2}}^{2} .
$$

Integrating (18) over $\Omega_{1}$ we obtain

$$
A_{1}\|f\|_{L^{2}(\Omega)}^{2} \leq \sum_{j=1}^{k}\left\|f_{j}\right\|_{L^{2}\left(\Omega_{1}\right)}^{2} \leq A_{2}\|f\|_{L^{2}(\Omega)}^{2} .
$$

This implies (8) with $C_{j}=k \cdot A_{j}, j=1,2$. To show the uniqueness of the decomposition (7) observe that any such decomposition must satisfy the linear system (17), whose non-singularity has been ensured by our choice of the $a_{j}$.

We can now complete the proof of our main result.

Proof of Theorem 1. Let $f \in L^{2}(\Omega)$. By Lemma 2 we can write $f$ as in (7). Since the $f_{j}$ are $\Lambda$-periodic and are in $L^{2}$ of any almost fundamental domain $D$ of $\Lambda$ it follows that we can expand each $f_{j}$ in the frequencies of $\Lambda^{*}$ (the dual lattice of $\Lambda$ )

$$
f_{j}(x)=\sum_{\lambda^{*} \in \Lambda^{*}} f_{j, \lambda^{*}} e\left(\lambda^{*} \cdot x\right), j=1,2, \ldots, k,
$$

with

$$
\left\|f_{j}\right\|_{L^{2}(D)}^{2}=\sum_{\lambda^{*} \in \Lambda^{*}}\left|f_{j, \lambda^{*}}\right|^{2}
$$

since the exponentials $e\left(\lambda^{*} \cdot x\right), \lambda^{*} \in \Lambda^{*}$, form an orthogonal basis of $L^{2}(D)$ (we assume without loss of generality that $|D|=1)$.

The completeness of (6) follows from (7):

$$
f(x)=\sum_{j=1}^{k} \sum_{\lambda^{*} \in \Lambda^{*}} f_{j, \lambda^{*}} e\left(\left(a_{j}+\lambda^{*}\right) \cdot x\right) .
$$

The fact that (6) is a Riesz sequence follows from (8):

$$
\frac{k}{C_{2}} \sum_{j, \lambda^{*}}\left|f_{j, \lambda^{*}}\right|^{2} \leq\left\|\sum_{j, \lambda^{*}} f_{j, \lambda^{*}} e\left(\left(a_{j}+\lambda^{*}\right) \cdot x\right)\right\|_{L^{2}(\Omega)}^{2} \leq \frac{k}{C_{1}} \sum_{j, \lambda^{*}}\left|f_{j, \lambda^{*}}\right|^{2} .
$$

As is clear from the proof above, the $k$-tuples of vectors $a_{1}, \ldots, a_{k}$ that appear in Theorem 1 are a generic choice: almost all $k$-tuples will do. The exceptional set in $\mathbb{R}^{d k}$ is a set of lower dimension.

With a little more care one can see that one can choose the vectors $a_{1}, \ldots, a_{k}$ to depend on $\Lambda$ and $k$ only and not on $\Omega$. In the proof of Lemma 2 the $a_{j}$ were chosen to ensure that the trigonometric polynomials (15) are all non-zero. Fix $\Lambda$ and $k$ and form the set of all polynomials of the form (15) which are not identically zero. This set of polynomials is countable and each such polynomial vanishes on a set of codimension at least 1 in $\mathbb{R}^{d k}$. It follows that the union of their zero-sets cannot possibly exhaust $\mathbb{R}^{d k}$ and we only have to choose the $a_{j}$ to avoid that union.

Thus there is a choice of $a_{j}$ that works for all $\Omega$ of the same lattice. This proof does not give uniform values for the constants $C_{1}$ and $C_{2}$ in (8) though. 


\section{REFERENCES}

[1] U. Bolle, On multiple tiles in $E^{2}$, Intuitive geometry (Szeged, 1991), Colloq. Math. Soc. János Bolyai, vol. 63, North-Holland, Amsterdam, 1994, pp. 39-43. MR.1383609 (97e:52032)

[2] Bálint Farkas, Máté Matolcsi, and Péter Móra, On Fuglede's conjecture and the existence of universal spectra, J. Fourier Anal. Appl. 12 (2006), no. 5, 483-494, DOI 10.1007/s00041-0055069-7. MR2267631 (2007h:52022)

[3] Bálint Farkas and Szilárd Gy. Révész, Tiles with no spectra in dimension 4, Math. Scand. 98 (2006), no. 1, 44-52. MR2221543 (2007g:52016)

[4] Bent Fuglede, Commuting self-adjoint partial differential operators and a group theoretic problem, J. Functional Analysis 16 (1974), 101-121. MR0470754 (57 \#10500)

[5] Nick Gravin, Sinai Robins, and Dmitry Shiryaev, Translational tilings by a polytope, with multiplicity, Combinatorica 32 (2012), no. 6, 629-649, DOI 10.1007/s00493-012-2860-3. MR.3063154

[6] Sigrid Grepstad and Nir Lev, Multi-tiling and Riesz bases, arXiv preprint arXiv:1212.4679 (2012).

[7] Alex Iosevich, Nets Hawk Katz, and Terry Tao, Convex bodies with a point of curvature do not have Fourier bases, Amer. J. Math. 123 (2001), no. 1, 115-120. MR1827279 (2002g:42011)

[8] Alex Iosevich, Nets Katz, and Terence Tao, The Fuglede spectral conjecture holds for convex planar domains, Math. Res. Lett. 10 (2003), no. 5-6, 559-569, DOI 10.4310/MRL.2003.v10.n5.a1. MR2024715(2004i:42020)

[9] Mihail N. Kolountzakis and Máté Matolcsi, Complex Hadamard matrices and the spectral set conjecture, Collect. Math. Vol. Extra (2006), 281-291. MR2264214(2007h:52023)

[10] M. N. Kolountzakis, On the structure of multiple translational tilings by polygonal regions, Discrete Comput. Geom. 23 (2000), no. 4, 537-553, DOI 10.1007/s004540010014. MR.1753701 (2001c:52025)

[11] Mihail N. Kolountzakis, The study of translational tiling with Fourier analysis, Fourier analysis and convexity, Appl. Numer. Harmon. Anal., Birkhäuser Boston, Boston, MA, 2004, pp. 131-187. MR2087242 (2005e:42071)

[12] Mihail N. Kolountzakis and Máté Matolcsi, Tiles with no spectra, Forum Math. 18 (2006), no. 3, 519-528, DOI 10.1515/FORUM.2006.026. MR2237932(2007d:20088)

[13] Gady Kozma and Shahaf Nitzan, Combining Riesz bases, arXiv preprint arXiv:1210.6383 (2012).

[14] Yurii I. Lyubarskii and Alexander Rashkovskii, Complete interpolating sequences for Fourier transforms supported by convex symmetric polygons, Ark. Mat. 38 (2000), no. 1, 139-170, DOI 10.1007/BF02384495. MR1749363 (2001m:32013)

[15] Yurii I. Lyubarskii and Kristian Seip, Sampling and interpolating sequences for multibandlimited functions and exponential bases on disconnected sets, J. Fourier Anal. Appl. 3 (1997), no. 5, 597-615, DOI 10.1007/BF02648887. Dedicated to the memory of Richard J. Duffin. MR1491937 (99f:30007)

[16] Jordi Marzo, Riesz basis of exponentials for a union of cubes in $\mathbb{R}^{d}$, arXiv preprint math/0601288 (2006).

[17] Basarab Matei and Yves Meyer, Quasicrystals are sets of stable sampling (English, with English and French summaries), C. R. Math. Acad. Sci. Paris 346 (2008), no. 23-24, 12351238, DOI 10.1016/j.crma.2008.10.006. MR2473299 (2010g:94050)

[18] Basarab Matei and Yves Meyer, Simple quasicrystals are sets of stable sampling, Complex Var. Elliptic Equ. 55 (2010), no. 8-10, 947-964, DOI 10.1080/17476930903394689. MR:2674875 (2011k:42019)

[19] Alexander Olevskii and Alexander Ulanovskii, On multi-dimensional sampling and interpolation, Anal. Math. Phys. 2 (2012), no. 2, 149-170, DOI 10.1007/s13324-012-0027-4. MR2917231

[20] Terence Tao, Fuglede's conjecture is false in 5 and higher dimensions, Math. Res. Lett. 11 (2004), no. 2-3, 251-258, DOI 10.4310/MRL.2004.v11.n2.a8. MR2067470 (2005i:42037)

Department of Mathematics and Applied Mathematics, University of Crete, GR-700

13, Heraklion, Crete, Greece

E-mail address: kolount@math.uoc.gr 